\title{
GLOBAL RESULTS IN CONTROL THEORY WITH APPLICATIONS TO UNIVALENT FUNCTIONS
}

\author{
BY S. FRIEDLAND ${ }^{1}$ AND M. SCHIFFER ${ }^{2}$
}

Communicated by R. T. Seeley, January 29, 1976

1. A problem in control theory. Many classical coefficient problems in the theory of univalent functions can be stated as the following control problem. Consider a first order differential system

$$
d x / d t=f(x, u(t)),
$$

where $x=\left(x_{1}, \ldots, x_{n}\right), u=\left(u_{1}, \ldots, u_{m}\right)$ and $f(x, u)=\left(f_{1}(x, u), \ldots\right.$, $\left.f_{n}(x, u)\right)$ are real valued vectors. Assume that $f$ is continuous on $R^{n} \times R^{m}$ and for fixed $u, f \in C^{1}\left(R^{n}\right)$. The values of $u(t)$ are in a compact domain $U \subset R^{m}$. Denote by $F$ the class of all piecewise continuous functions $u(t)$ for $t \geqslant 0$ with the values in $U$. Let $x(t)$ satisfy a fixed initial condition $x(0)=\xi$. Denote by $x(t, u)$ the solution of the system above for a given $u(t)$ in $F$. Let $F(x)=$ $F\left(x_{1}, \ldots, x_{n}\right)$ belong to $C^{1}\left(R^{n}\right)$.

THEOREM 1. Let $u^{*}=u^{*}(t)$ be a solution of the problem $\sup _{F} F(x(T, u))$ $=F\left(x\left(T, u^{*}\right)\right)$, for $T>0$. Consider the system

$$
d x / d t=f\left(x, u^{*}(t)\right), x(\tau)=\eta
$$

for $0 \leqslant \tau \leqslant T$. Define a function $F_{\tau}$ by the equality $F_{\tau}(\eta)=F(x(T))$. Then $x\left(\tau, u^{*}\right)$ solves the problem $\sup _{F} F_{\tau}(x(\tau, u))=F_{\tau}\left(x\left(\tau, u^{*}\right)\right)$.

The proof of the theorem follows by considering the functions $u(t)$ such that $u(t)=u^{*}(t)$ for $\tau<t \leqslant T$. In case where $f(x, u)=A(u) x$ and $F(x)=$ $\lambda_{0}^{\prime} x \quad$ Theorem 1 has a very simple form. Here $A(u)=\left(a_{i j}(u)\right)_{1}^{n}$ and $a_{i j}(u) \in$ $C\left(R^{m}\right)$. By $A^{\prime}$ and $\lambda^{\prime}$ we denote the corresponding transposed matrix and vector.

THEOREM 2. Consider a control system $d x / d t=A(u(t)) x$. Let $u^{*}(t)$ solve the linear problem

$$
\sup _{F} \lambda_{0}^{\prime} x(T, u)=\lambda_{0}^{\prime} x\left(T, u^{*}\right) .
$$

Then $x\left(\tau, u^{*}\right)$ solves the linear problem

$$
\sup _{F} \lambda^{\prime}(\tau) x(\tau, u)=\lambda^{\prime}(\tau) x\left(\tau, u^{*}\right)
$$

AMS (MOS) subject classifications (1970). Primary 49B10; 30A34.

${ }^{1}$ The first author was supported in part by NSF grant MPS 72-05055 A02.

${ }^{2}$ The second author was supported by NSF grant MPS 75-23332. 
$0<\tau<T$, where $\lambda(t)$ is the solution of $d \lambda / d t=-A^{\prime}\left(u^{*}\right) \lambda, \lambda(T)=\lambda_{0}$.

2. Univalent functions. Denote by $S$ the set of all analytic univalent functions $f(z)=z+\Sigma_{k=2}^{\infty} a_{k} z^{k}$ in the unit disc $D$. Let $f$ be a slit function, i.e. $f$ maps $D$ onto a slit domain. According to Loewner [1] $f$ can be embedded in a semigroup of univalent functions $g(z, t)=e^{t}\left(z+\Sigma_{k=2}^{\infty} a_{k}(t) z^{k}\right)$ which satisfies the equation

$$
\frac{\partial g}{\partial t}=z \frac{\partial g}{\partial z} \frac{1+e^{i \varphi(t)} z}{1-e^{i \varphi(t)} z}, \quad g(z, 0)=f(z) .
$$

Here $\varphi(t)$ is a real piecewise continuous function for $t \geqslant 0$. Denote by $A_{n}$ the set of vectors $a^{(n)}=\left(a_{1}, \ldots, a_{n}\right),\left(a_{1}=1\right)$ which are the first $n$ coefficients of some $f$ in $S$. Let $f(z, t)=e^{-t} g(z, t)=z+\Sigma_{k=2}^{\infty} a_{k}(t) z^{k}$. Then $a^{(n)}(t)$ satisfies the system

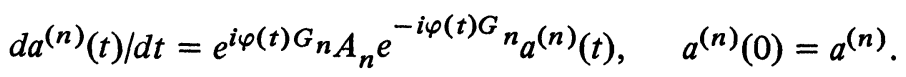

Here $A_{n}=\left(a_{k j}\right)_{1}^{n}$ and $G_{n}=\left(d_{k} \delta_{k j}\right)_{1}^{n}$ are the matrices: $a_{k j}=0$ for $j>k, a_{k k}=$ $k-1, a_{k j}=2 j$ for $j<k, d_{k}=k-1, k, j=1, \ldots, n$. The following result is basic for applications of the method of control theory to coefficient problems for univalent functions.

THEOREM 3. Let $\varphi(t)$ be a real measurable function for $t \geqslant 0$. Consider the system $d a^{(n)} / d t=-e^{i \varphi(t) G_{n}} A_{n} e^{-i \varphi(t) G_{n}} a^{(n)}$ for $t \geqslant 0$.

Then (i) $A_{n}$ is invariant under the flow defined by the system above. That is, if $a^{(n)}(0) \in A_{n}$ then $a^{(n)}(t) \in A_{n}$ for any $t>0$.

(ii) Let $\alpha^{(n)} \in A_{n}$ and consider the set of all possible paths $a^{(n)}(t)$ starting from the point $\alpha^{(n)}$ for all choices of $\varphi$. Then this set is dense in $A_{n}$.

Let $a_{*}^{(n)}=\left(a_{1}^{*}, \ldots, a_{n}^{*}\right)$ be a boundary point of $A_{n}$. According to [2] , the corresponding function $f^{*}(z)=z+\Sigma_{k=2}^{\infty} a_{k}^{*} z^{k}$ is a slit function. So $f^{*}(z)$ generates the corresponding $\varphi^{*}(t)$ which appears in the Loewner equation. Using Theorems 2 and 3, we obtain

THEOREM 4. Let $a_{*}^{(n)}$ solve the problem

$$
\max _{\mathrm{A}_{n}} \operatorname{Re}\left\{\sum_{k=1}^{n} \lambda_{k}^{0} a_{k}\right\}=\operatorname{Re}\left\{\sum_{k=1}^{n} \lambda_{k}^{0} a_{k}^{*}\right\}
$$

subject to $m$ constraints $a_{k}=a_{k}^{*}, k=1, \ldots, m,(m \leqslant n-1)$. Let $a^{(n)}(t)$ be generated by the Loewner equation

$$
d a^{(n)} / d t=e^{i \varphi^{*}(t) G_{n}} A_{n} e^{-i \varphi^{*}(t) G_{n}} a^{(n)}, \quad a^{(n)}(0)=a_{*}^{(n)} .
$$

Define $\lambda^{(n)}(t)$ to be

$$
d \lambda^{(n)} / d t=-e^{-i \varphi^{*}(t) G}{ }_{n} A_{n}^{\prime} e^{i \varphi^{*}(t) G} n \lambda^{(n)}, \quad \lambda^{(n)}(0)=\lambda_{0}^{(n)} .
$$


Then $a^{(n)}(t)$ solves the problem

$$
\begin{aligned}
\max _{A_{n}} \operatorname{Re}\left\{\sum_{k=1}^{n} \lambda_{k}(t) a_{k}\right\} & =\operatorname{Re}\left\{\sum_{k=1}^{n} \lambda_{k}(t) a_{k}(t)\right\} \\
a_{k} & =a_{k}(t), k=1, \ldots, m, \text { for } t>0 .
\end{aligned}
$$

In particular we obtain that if $a_{*}^{(n)}$ is a supporting point of $A_{n}$, so is $a^{(n)}(t)$ for $t>0[2,10.3]$. Let $\xi^{k}=\left(0, \ldots, 0,1, \xi_{k+2}^{k}, \ldots, \xi_{n}^{k}\right)$ and $\eta^{k}=$ $\left(\eta_{1}^{k}, \ldots, \eta_{k}^{k}, 1,0, \ldots, 0\right), k=0, \ldots, n-1$, where

$$
\xi_{r}^{k}=(-1)^{k}\left(\begin{array}{c}
r+k \\
r-k-1
\end{array}\right), \eta_{r}^{k}=(-1)^{r-1} \frac{r}{k+1}\left(\begin{array}{c}
2 k+2 \\
k-r+1
\end{array}\right) .
$$

Theoerm 5. Assume that the Koebe function $K(z)=z /(1-z)^{2}$ solves a linear problem

$$
\max _{A_{n}} \operatorname{Re}\left\{\lambda^{(n)^{\prime}} a^{(n)}\right\}=\operatorname{Re}\left\{\lambda^{(n)^{\prime}} e^{(n)}\right\}
$$

where $e^{(n)}=(1,2, \ldots, n)$.

Then the Koebe function also satisfies $\max _{A_{n}} \operatorname{Re}\left\{\lambda^{(n)}(x)^{\prime} a^{(n)}\right\}=$ $\operatorname{Re}\left\{\lambda^{(n)}(x)^{\prime} e^{(n)}\right\}$, for $0<x<1$, where $\lambda^{(n)}(x)=\sum_{r=0}^{n-1} x^{r}\left(\lambda^{(n)^{\prime}} \xi^{r}\right) \eta^{r}$. In particular, if $\operatorname{Re}\left\{a_{n}\right\} \leqslant n$, then

$$
\begin{aligned}
& \operatorname{Re}\left\{\sum_{r=0}^{n-1}(-1)^{r} x^{r}\left(\begin{array}{c}
n+r \\
n-r-1
\end{array}\right)\left[\left(\eta^{r}\right)^{\prime} a^{(n)}\right]\right\} \\
& \quad \leqslant \operatorname{Re}\left\{\sum_{r=0}^{n-1}(-1)^{r} x^{r}\left(\begin{array}{c}
n+r \\
n-r-1
\end{array}\right)\left[\left(\eta^{r}\right)^{\prime} e^{(n)}\right]\right\}, \quad \text { for } 0<x<1 .
\end{aligned}
$$

Thus, from $\operatorname{Re}\left\{a_{4}\right\} \leqslant 4$ we obtain the inequality

$$
\begin{aligned}
\operatorname{Re}\left\{x^{2} a_{4}\right. & \left.+6 x(1-x) a_{3}+2\left(7 x^{2}-12 x+5\right) a_{2}\right\} \\
& \leqslant 14 x^{2}-30 x+20
\end{aligned}
$$

for $0<x<1$. The full details and the proofs will appear elsewhere.

\section{REFERENCES}

1. K. Loewner, Untersuchungen über schlichte konforme Abbildungen des Einheitskreises. I, Math. Ann. 89 (1923), 103-121.

2. A. C. Schaeffer and D. C. Spencer, Coefficient regions for schlicht functions, Amer. Math. Soc. Colloq. Publ., Vol. 35, Amer. Math. Soc., New York, 1950. MR 12, 326.

SCHOOL OF MATHEMATICS, THE INSTITUTE FOR ADVANCED STUDY, PRINCETON, NEW JERSEY 08540

THE HEBREW UNIVERSITY, JERUSALEM, ISRAEL

DEPARTMENT OF MATHEMATICS, STANFORD UNIVERSITY, STANFORD, CALIFORNIA 94305 